\title{
La reforma administrativa de los sesenta y la transición democrática ${ }^{1}$
}

Kim Helsvig *

\section{Administración para el desarrollo económico}

Desde mi punto de vista, la reforma administrativa, iniciada en 1957, indujo un proceso que facilitó la disolución del régimen franquista al afectar no sólo la cultura políica e intelectuál sino también la política en sí. La historia de esta reforma y la subsecuente transición a la democracia es no obstante la historia de una serie de consecuencias no intencionadas. Con el objeto de entender la dinámica de este proceso debemos remontarnos a diciembre de 1956 cuando Laureano López Rodó fue designado al frente de la recién creada Secretaría General Técnica de la Presidencia del Gobierno. Su primera iniciativa fue la elaboración del Informe Acerca de la Reorganización de la Administración Central del Estado que fue presentado a Carrero Blanco y Franco. En este informe López Rodó se mostraba a favor de la hipótesis de que la mayoría de los problemas del régimen en ese momento se debían a la ineficacia de la Administración Pública, y lo que se necesitaba sobre todo era una reforma administrativa total. Este punto de vista de un joven profesor de Derecho administrativo que se incorpora al nexo del poder político puede no resultar sorprendente, pero creo que debemos buscar más allá de intereses estrictamente profesionales si queremos entender su proyecto. Antes de todo, creo que debemos tomar en serio a lópez Rodó cuando dice que cree firmemente en la fuerza formativa de la ley: Siempre he creído en la ley [...] Configura la realidad presente y futura del país [...] Lo diré otra vez: la fuerza formativa de la ley es una de mis más firmes conviccio- nes $^{2}$. Esta fuerza formativa tenía que involucrarse en un proceso de cambio social que escapaba claramente de los límites del formalismo jurídico. Durante un discurso ante las Cortes el 15 de julio de 1958, LOPEZ RODO dijo:

El Derecho mismo no puede concebirse ya como mera for$\mathrm{ma}$, sino como eficaz instrumento de justicia. $Y$ la justicia en materia económico-social no se consigue con sólo dictar normas jurídicas, sino uniendo a ellas una eficaz acción que estimule y complete la iniciativa privada [.. . . Sin una acción administrativa decidida, homogénea, rápida [.. l las mismas garantías jurídicas resultarían insuficientes.l.. Hoy en día tiende a superarse el excesivo ordenancismo estatal y a montar la Administración con criterios de eficacia, y esto exige el conocimiento a fondo de problemas económicos, administrativos y de organizacion que exceden del campo estrictamente jurídico. ${ }^{3}$.

Así, la reforma administrativa propuesta por Lopez RoDó implicaba una concepción del papel de la ley que se apartaba de raíz del enfoque tradicional español al respecto. Para la concepción de LOPEZ RoDo, la ley debía ser sobre todo un instrumento cientííico social para dirigir el cambio social, y no entenderse como el garante de derechos más o menos eternos. En 1963 dijo: $\bullet$ A mi modo de ver, pueden considerarse hoy fines primordiales del Estado el desarrollo económico y la igualdad de oportunidades como fundamentos del progreso social. ${ }^{4}$. Crecimiento económico y progreso social eran interpretados como una garantía contra protestas y desórdenes sociales, y LOPEZ RODO sostenía que en esto se basaba en evidencias científicas ${ }^{5}$. De esta manera, la reforma administrativa se presentaba como una herramienta para lograr el desarrollo económico y la estabilidad social. 
Aunque la propuesta de LOPEZ RODÓ de una reforma administrativa tenía un importante sector en contra, tanto dentro de la coalición franquista como en la Administración pública, el joven profesor fue capaz de convencer a Franco de la viabilidad del proyecto. Éste no es el lugar para analizar la compleja lucha por el poder dentro de la coalición franquista que finalmente arrojó a la arena política española a los denominados tecnócratas $^{6}$. Solamente voy a señalar el hecho de que en medio del turbulento clima político y social de los años 1956 y 1957, LOPEZ RoDo fue capaz de presentar argumentos tanto "neutrales" como - científicos para legitimar el régimen a la vez haciéndolo un instrumento para sus propias aspiraciones políticas, al sostener que en el estado de transición de una economía hacia una etapa de desarrollo sostenido se necesita una autoridad política que ponga en funcionamiento las medidas técnicamente correctas sin tener que sufrir un atomizante e 'irracional' debate político. Durante una conferencia en la Universidad de Santiago de Compostela en septiembre de 1956 afirmaba que;

-Todas las grandes reformas administrativas se han realizado a la sombra de una gran figura política. Y la labor administrativa ha sido lo que ha perdurado a través de los cambios políticos posteriores [.. I Nada queda hoy del Imperio napoleónico, pero ahí están todavía los Prefectos y el Consejo de Estado dando testimonio vivo y actual de su paso por la historia de Francia." .

y después decía:

¿En su bien conocido ensayo Stages of Economic growth, Rostow destaca la presencia de un hombre extraordinario que sabe cómo catalizar las energías latentes del pueblo, devolviéndole la confianza en sí mismo, como uno de los factores desisivos para el despegue económico. Debe reconocérsele al Caudillo el mérito de que los españoles hayamos recuperado la confianza en nosotros mismos." ${ }^{8}$.

Así, en la borrascosa situación de fines de los cincuenta, LO. PEZ RODO consiguió establecer una relación cuasi instrumental entre la reforma administrativa de los tecnócratas para el desarollo económico y el rol histórico de Franco a quien debía reconocérsele el mérito de que los españoles ahayamos recuperado la confianza en nosotros mismos.

La reforma propuesta tenía tres aspectos: el legal, el funcional y el cultural. El aspecto legal tenía que institucionalizarse a través de la implementación de leyes que implicaran especialización, desconcentración y centralización ejecutiva". El aspecto funcional debía alcanzarse mediante el entrenamiento en nuevas técnicas de procedimientos en el Centro de Formación y Perfeccionamiento de Funcionarios. Por último, el aspecto cultural debía canalizarse a través del aprendizaje en el espíritu del mismo Centro, así como la creación de la revista Documentación Administrativa.

\section{La Escuela de Alcalá en el} centro de la lucha burocrática y política

El Centro de Formación y Perfeccionamiento de Funcionarios fue establecido el 22 de septiembre 1958 como institucionalización de los cursos y conferencias que se habian estado desarrollando bajo el gabinete de Estudios Administrativos durante más de un año ${ }^{0 .}$. Al comienzo, hasta mayo de 1960, la institución estaba situada en el centro de Madrid, en la calle Trafalgar, pero más tarde fue transferida a los majestuosos edificios del antiguo convento que había ocupado la antigua Universidad de Alcalá en Alcalá de Henares. Durante el período que he estudiado, entre fines de 1958 y 1970, un total de 15.455 personas fueron educadas o entrenadas en el centro ". Este número incluye todos aquellos que asistieron a cursos antes de que el programa para los Técnicos se creara en 1960. Parece ser que la mayoría de los asistentes a cursos en los primeros dos años eran funcionarios civiles superiores con autoridad ejecutiva, ya que sólo algo más de un veinte por ciento no tenía grado universitario $^{12}$. Si un cálculo aproximado permite suponer que al menos el cincuenta por ciento de aquellas personas entrenadas en el centro antes de su reubicación en Alcalá en 1960 eran funcionarios civiles superiores, entonces durante todo el período, desde 1958 hasta 1970, cerca de 4.500 funcionarios civiles superiores o se educaron o realizaron entrenamiento posterior en la Escuela de Alcalá ${ }^{13}$.

El Centro fue definido desde el comienzo como un receptor de ideas foráneas sobre cómo llevar adelante una administración moderna, tal como se afirma en el informe anual de 1958: uno de los principales objetivos del Centro era $\cdot[. . \mid$ conocer nuevos métodos y experiencias y de confrontarlos a los nacionales $\left[. .\left.\right|^{14}\right.$. . La necesidad percibida de "confrontar" los métodos y experiencias nacionales refleja el intento de desafiar el poder de los poderosos "Cuerpos especiales" dentro de la Administración. Estos Cuerpos tenían una forma de reclutamiento propia y no realizaron entrenamiento en el nuevo Centro. Éste, por su parte, se concentró en la educación y el perfeccionamiento de los llamados "Cuerpos generales". Así nació dentro del Centro un nuevo y poderoso Cuerpo general, el llamado Técnico de Administración Civil. La intención era que éste tuviera carácter ejecutivo dentro de cada departamento siendo al mismo tiempo un Cuerpo interministerial que debía trabajar por la coordinación en conjunto. El Cuerpo Técnico de Administración Civil debía representar "[... un paso decisivo hacia la uniformación de los Cuerpos ${ }^{15}$. Y esto fue percibido por los llamados . Cuer- 
pos especiales" como una agresión dirigida contra su hegemonía tradicional. Miguel Beltrán escribe:

- La creación de dichos Cuerpos, que objetivamente supuso una notable modernización de la dividida y obsoleta Administración estatal de la época, desencadenó, pues, una auténtica lucha por el poder burocrático, tanto entre Cuerpos (y en concreto entre el Técnico de Administración Civil y los "especiales" de titulación superior de cada Ministerio), como entre Ministerios: el de Hacienda, adalid de las pautas más tradicionales, frente al de la Presidencia, impulsor de la reforma. Y todo ello además, y al margen, de la lucha por el poder político que la reforma de la Administración implicaba, sin duda, para el grupo "tecnocrático". ${ }^{16}$.

Por lo tanto, es razonable concluir que la que sería más tarde la Escuela Nacional de Administración Pública, o simplemente La Escuela de Alcalá, desde el primer momento se encontró en medio de una feroz lucha burocrática y política por el poder. La arbitrariedad y dispersión tradicionales eran confrontadas por el grupo tecnocrático y sus esfuerzos por crear un nuevo tipo de Administración para el desarrollo económico a través del nuevo Centro.

\section{Conferencias y contactos internacionales}

En todo momento la Escuela se mostró extremadamente receptiva a las influencias foráneas. Era como si la organización administrativa extranjera fuera una especie de tecnología con fundamento científico aplicable directamente a la realidad española. Durante 1957 LOPEZ RODO conoció politólogos y administradores de EEUU, RU y Francia para, por asi decirlo, beber en las fuentes de la ciencia y tecnología administrativa internacionales. Durante una conferencia en la Escuela de Economía de Londres, el 7 de noviembre de 1957, LOPEZ RODO dijo que: "Las instituciones politicas pueden variar de un país a otro, debido a sus diferentes características, pero las instituciones y actividades administrativas son, por el contrario, un factor común en la vida de las naciones. ${ }^{17}$. No obstante, parece claro que la más inmediata fuente de inspiración de la Escuela de Alcalá fue la École Nationale d'Administration de Francia. En el informe anual de 1959 se lee que dadas las características de nuestro régimen administrativo, resulta indiscutible la utilidad que tiene el conocimiento y estudio de la Administración francesa, ya que es la que presenta una estructura y problemática más afín a la Administración española" ${ }^{18}$.

Durante la década del sesenta la Escuela de Alcalá fue sede de varias conferencias internacionales relativas al trabajo admi- nistrativo y el desarrollo económico. Durante estas conferencias participaron personalidades como Ernst FORSTHOFF (Rectsstaatlichkeit und Sozialstaatlichkeit: anfsätze und erfäbrungen), Georges LANGROD (The International civil service: its origins, its nature, its evolution), Fritz MORSTEIN MARX (The administrative state: an introduction), Albert WaTERSTON (Development planning: lessons of experience), Alfred Sauvy (Histoire economique de la France), Brian Crozier (The minimum state: beyond party politics), Pierre BAUCHET (Economic Planning: the French experience), y W.W. Rostow (The stages of economic growth, A noncommunist manifesto).

La teoría de las etapas del crecimiento económico formulada por Rostow ${ }^{19}$ tuvo profunda influencia sobre la percepción por parte de LOPEZ RODO de la relación entre administración y desarrollo económico. Rostow es varias veces aludido en su autobiografía, Memorias. Tanto LOPEZ RODÓ como ROsTOw postulaban la necesidad de contener el comunismo en una fase de desarrollo económico crucial, y compartían ciertas teorías respecto de cómo conseguir este objetivo. Rostow, como Director de Políticas y Planeamiento del Departamento de Estado de los EEUU durante la Administración Kennedy y asesor principal del presidente Johnson en Vietnam, por un lado ${ }^{20}$, y LOPEZ RODO como político clave dentro del ferozmente anticomunista aparato franquista, por el otro. De esta manera, LOPEZ RODó utilizó a Rostow como aval científico para la teoría de la necesidad de un Estado autoritario en esa etapa peculiar del desarrollo económico de España (precondiciones para el despegue), y por lo tanto se lo puede ligar de manera estrecha a la relación entre las reformas y la dictadura.

Los tecnócratas estaban, por lo general, a favor de permitir la participación de España en el proceso - ya en marcha - de integración europea. El segundo grupo de técnicos de la Escuela de Alcalá tuvo siete conferencias en 1962 sobre los temas Desarrollo bistórico doctrinal de la unidad entropea e Integridad entropea ${ }^{21}$. Acerca del Comisario del plan de desarrollo Francés, Pierre Masu, LOPEZ RODO escribió en 1963 que: "La planificación nacional l. . I no es más que una etapa necesaria, pero transitoria, hacia una planificación plurinacional en el seno de grandes conjuntos económicos regionales ${ }^{22}$. "Esto se relaciona con la solicitud española, en 1962, de ingreso en la que más tarde sería la Unión Europea.

\section{Selección y educación del Cuerpo Técnico de Administración Civil}

Los candidatos tenían que ser seleccionados tras pasar tres tests eliminatorios. El primer año se registraron un total de 1.056 
solicitudes de ingreso para un total de 70 vacantes dentro de los Ministerios. 470 aprobaron el primer examen que trataba sobre Lengua. El segundo test consistía en una evaluación escrita de cuatro horas sobre uno de los temas: Economía, Historia Política, Derecho Administrativo o Ciencia Política. Durante el test final oral el candidato tenía que responder a preguntas relacionadas con los siguientes temas: Historia Social y Política de España y el resto del mundo, organización del Estado, economía, finanzas públicas, Derecho administrativo y ciencia administrativa. En el Boletín Oficial del Estado el programa del test final era presentado mediante 133 puntos relacionados con estos temas. 46 de ellos se relacionaban con las ciencias administrativas y abarcaban desde conocimiento de la nueva organización formal de la Administración hasta técnicas de racionalización para ser aplicadas dentro de determinados departamentos ${ }^{23}$. Por lo tanto, una gran parte del currículum para el examen final de ingreso giraba alrededor de las dos leyes básicas que afectaban la reforma: la Ley de Régimen Jurídico de la Administración del Estado, relativa a la reforma organizacional, y la Ley de Procedimiento Administrativo, respecto de los nuevos procedimientos a aplicarse dentro de la Administración.

Al estudiar los exámenes de ingreso mencionados se advierte el esfuerzo en dirigir la orientación del funcionario público hacia una actitud menos formalista y más inclinada a la innovación, lo cual coincide con el proceso de ruptura general con el formalismo jurídico tradicional, todo lo cual haría de los técnicos una suerte de "ingenieros sociales" caracterizados por $\cdot[\ldots]$ un espíritu innovador y dinámico lejos de la asepsia formalista en que parecían configurarlo las viejas formulas ${ }^{24}$. Miguel BeLTRÁN afirma que uno de los rasgos especiales de la educación en Alcalá era que se orientaba a permitir que las normas de la ley fueran en si un factor de transformación, a diferencia de la actitud tradicional que las colocaba en el rol de garante de privilegios o un elemento importante en la conservación del statu quo. Como tal la Escuela se inspiró en buena medida en el pragmatismo jurídico anglosajón, en concreto el norteamericano ${ }^{25}$. Por ello la Escuela enseñaba técnicas de ajuste de presupuesto en vez de dictar cátedra sobre Derecho Financiero, Administración de Personal o en vez de tratar aspectos jurídicos de la Administración pública, Economía, Sociología, Ciencia Política e Historia reemplazando el Derecho Administrativo y constitucional ${ }^{26}$.

En el plan de estudios para el primer grupo de técnicos, entre 1960 y 1961, se afirma que una parte importante de la educación impartida tendrá como meta $\cdot[. .$.$] promover una$ mentalidad creadora y administrativa y un propósito dinámico en su futura actuación ${ }^{27}$. Al revisar algunas de las conferencias durante el segundo curso en 1961 y 1962 , salta a la vista que estamos frente a lo que hemos denominado administración para el desarrollo económico. En doce conferencias el tema era precisamente la planificación del desarrollo económico, como por ejemplo en la ponencia de Enrique Fuentes Quintana la planificación del desarollo económico y la política fiscal. Otras ponencias tenían títulos como los siguientes: Desarrollo económico social, La planificación y sils objetivos, Aspectos sociales de la planificación económica, Modelos econométricos de la planificación económica, La impulsión de la acción administrativa y desarrollo económico de los Gobiemos Civiles, Seguridad Social y Desarrollo económico, La planificación del desarrollo económico y los transportes, La planificación del desarrollo económico y la agricultura ${ }^{28}$.

El programa general a mediados de los sesenta consistía en un total de 298 horas de conferencias divididas en las siguientes temáticas: 1) Organización y Métodos, 2) Administración de personal, 3) Procedimiento Administrativo, 4) Administración Financiera y Coste, 5) Psicosociologia Administrativa, 6) Estadística, 7) Politica Económica y 8) Estructura Económica.

Los cursos de Organización y Métodos versaban sobre nuevas técnicas para hacer los procesos administrativos más eficaces. Una parte de estos cursos trataba acerca de los problemas relacionados con la dirección de una organización girando siempre alrededor de conceptos tales como planeamiento, programación, coordinación y control. El punto central será la estructura organizativa como un factor de productividad técnica ${ }^{20}$. Otra parte de estos cursos de Organización y Métodos trataba sobre la simplificación de los procedimientos. En dicha parte el concepto clave sería la "organización científica del trabajo, explícitamente inspirado de TaYlor. Además, se analizan maneras de incrementar la eficacia a través de estudios de costo y tiempo ${ }^{30}$. Otra de las partes de esta asignatura se dedicaba al estudio y elaboración de procedimientos para la estandarización y clasificación de documentos. La parte final de estos cursos se extendía al análisis de la mecanización del trabajo administrativo ${ }^{31}$.

Los cursos sobre Administración del Personal enfatizaban las semejanzas entre la administración de una empresa comercial y la administración pública y la necesidad de concebir a la administración como una empresa con fundamento científico ${ }^{32}$. Durante estos cursos, se discutian problemas respecto de la flexibilidad de la Administración ante la rigidez de la Administración legislativa en relación a la Ley de Régimen Jurídico de la Administración del Estado. Además, se analizaba la Administración española desde una perspectiva comparada: los procedimientos tanto de función como de selección son vistos a la luz de experiencias similares en los EEUU, el Reino Unido, Francia, Alemania Occidental e Italia ${ }^{33}$.

Los cursos sobre Procedimiento Administrativo trataban en su totalidad sobre los principios de la Ley de Procedimiento Administrativo. En síntesis, estos cursos desarrollaban el tema de la racionalización de la Administración, y la manera en que los mencionados principios se integraban en la nueva ley ${ }^{34}$. 
Las lecciones sobre Administración Financiera y Coste eran un curso básico que, sobre todo, pretendía presentar los conceptos y problemas básicos relacionados con este tema. Se elaboraba sobre los aspectos políticos, jurídicos y económicos de la responsabilidad administrativa en cuanto a presupuestos ${ }^{35}$. Además, se hacía hincapié en el control, tanto interno como externo, de la Administración financiera y la manera de evaluar su impacto en la sociedad ${ }^{36}$.

El curso de Psicosociologia Administrativa se abría con una introducción a la metodología de la psicología y de la sociología experimentales y la utilidad de la aplicación de estas ciencias en organizaciones en general. Los conceptos y las actitudes dentro de la teoría de las "relaciones humanas" se presentan relacionados con los aspectos de la Administración pública que más se asemejan a la administración de una empresa. Luego, se sacaban conclusiones sobre la aplicabilidad de estas ciencias en el trabajo real de los técnicos. En la continuidad del mismo, se analizaba la problemática de la motivación y la actitud y las técnicas para el mejoramiento de éstas. En la última parte se trataban técnicas de relaciones públicas y sus efectos sociales, y la relevancia de utilizarlas dentro de la Administración pública ${ }^{37}$.

El curso de Estadística pone de relieve el papel de ésta dentro de la Organización y Métodos. Las lecciones estudiaban el muestreo de datos, la confección de cuestionarios y el análisis de los resultados. Se buscaba lograr que el estudiante conociera y fuera capaz de manejar conceptos gráficos y matemáticos mediante ejercicios prácticos ${ }^{38}$. La importancia de esta asignatura se reflejaba en la última etapa del programa de cursos: "Las estadisticas como la ciencia fundamental para actividades de planeamiento y control. ${ }^{39}$.

Durante los años sesenta se establecieron dos importantes Institutos de investigación en la Escuela. Primero, a partir de la orden de la Presidencia del Gobierno del 11 de septiembre de 1963, se creó el Instituto de Desarrollo Económico, un año después de la puesta en marcha del primer Plan de Desarrollo Económico y Social. Luego, en 1966, se estableció el Instituto de Estudios Administrativos ${ }^{40}$, que se convertiría pronto en el nexo con, entre otras actividades, la planificación de las reformas educativas, siendo dirigido por José Luis Villar Palasí. En 1969, éste fue nombrado Ministro de Educación y Ciencia, y como tal fue responsable de la implementación, al año siguiente, de la Ley General de Educación.

Ambos Institutos fueron creados como respuesta directa al clesarrollo de los procesos de reforma tecnocrática a gran escala, o sea, la reforma económica mediante el uso del planeamiento indicativo (Instituto de Desarrollo Económico) y la demanda de personal calificado para responder a las exigencias de una sociedad cada vez más tecnificada y compleja (Instituto de Estudios Administrativos). En el proceso de reforma general, los planes de desarrollo económico estaban en el centro del de- bate político en 1963 , de la misma forma que lo estuvo la necesidad de una reforma educativa a fines de los sesenta. De esta manera podemos distinguir el aspecto predominantemente instrumental de la Escuela de Alcalá dentro del proceso de modernización y 'europeización' del régimen. Desde mi punto de vista, las siguientes tres características concretan la esencia de la orientación intelectual de la Escuela y como tal el proceso de reformas en general, al menos en la etapa inicial: 1) Una aceptación casi incondicional de la relevancia de las experiencias en otros países, 2) La percepción de la educación en diferentes aspectos de la ciencia administrativa como un medio formal y mental para alcanzar el supremo objetivo del desarrollo económico, y 3) Una firme convicción de que la ciencia y la tecnología juegan el papel de instrumentos neutrales en este proceso.

Los cursos de formación de Técnicos de la Administración Civil tenían la misión de crear una nueva persona dentro de la Administración pública española: el ingeniero social no-formalista capacitado para administrar el cambio social y la eficiencia a través del conocimiento de la sociología administrativa, psicología de grupo y administración financiera para propósitos sociales definidos. Como se afirma en la Crónica de 10 años de 1970: 4...l, la Escuela de Alcalá constituye un capítulo vivo de esas "ciencias de comportamiento", las behavioral sciences anglosajonas, de tan honda influencia en la renovación contemporánea de las ciencias políticas y administrativas. "

\section{La Escuela de Alcalá y la ética profesional del Opus Dei}

No es mi intención presentar aquí la Escuela de Alcalá como un proyecto exclusivo del Opus Dei, pero de cualquier manera queda claro que su creación fue casi por completo un proyecto de López Rodó, numerario de sla obra. Su amigo personal y también numerario del Opus Dei, José Luis Meilán Gil, fue profesor de Administración económica en la Escuela ${ }^{42}$, y publicó siete libros en sus diferentes colecciones a lo largo de la década del sesenta ${ }^{43}$. En 1969 fue designado Secretario General Técnico de la Presidencia del Gobierno ${ }^{41}$, el mismo cargo que había ocupado López Rodó antes de ser nombrado Comisario del Plan de Desarrollo en 1962. José Luis Villar Palasí, también numerario del Opus Dei ${ }^{45}$, era profesor de Administración Financiera en la Escuela ${ }^{46}$ y fue designado Director del recién creado Instituto de Estudios Administrativos en $1966^{47}$. Encabezó desde 1969 el Ministerio de Educación y Ciencia. Otro numerario del Opus Dei, Javier Irastorza Revuelta, era Di- 
rector Adjunto del Instituto de Desarrollo Económico de la Escuela llegando en 1969 al cargo de Secretario General de la Comisaría del Plan de Desarrollo ${ }^{18}$. Aunque esta lista no está completa, se puede inferir que personas con vínculos estrechos con el Opus Dei ocuparon puestos clave en la Escuela, sin dejar de mencionar su importante presencia política dentro del régimen mismo.

Por otra parte, es importante resaltar el carácter elitista de la ideología del Opus Dei. Desde sus comienzos La Obra tenía la misión de trabajar para capturar una élite intelectual, a la cual se debía reorientar de acuerdo a los principios éticos sostenidos por el Opus Dei, los cuales así extenderían su influencia para servir de guía a la sociedad. En una carta de 1934 a Francisco Morán, Vicario de la Diócesis de Madrid, Escrivá de Balaguer, el fundador del Opus Dei, caracteriza sus actividades como aapostolado sacerdotal entre los intelectuales " ${ }^{49}$. En la Constitución del Opus Dei, elaborada en 1950, se establece que la actividad de la organización debe dirigirse hacia •los intelectuales y la vanguardia de la sociedad. Hoy en día en los estatutos se refiere a •[..) especialmente intelectuales ${ }^{50}$. El ex-miembro del Opus Dei Alberto Moncada dice que la Orden (Prelatura de la Santa Cruz y del Opus Dei) siempre tuvo una intención explícita de formar una suerte de earistocracia de la inteligencia. ${ }^{51}$, y Escrivá de Balaguer recordaba haber desarrollado la mayor parte de su actividad por claustros universitarios. Se ha llegado a sugerir que alguna vez su fundador consideró la posibilidad de llamar al instituto Sociedad de Cooperación Intelectual. El $\$ 202$ de la Constitución del Opus Dei de 1950 establece que ael medio más idóneo para el apostolado del Opus Dei es la función pública, y por sobre todo la que incluye dirección. ${ }^{52}$. Como tal, pienso que es justo suponer que varios miembros de la organización entendieron a la Escuela de Alcalá y la Administración pública como un medio ideal para su apostolado.

Tal como en la ética calvinista, el Opus Dei tiene una ética que valora profundamente el trabajo realizado en reste mundo. Con BorDiEu se puede decir que la conducta de los miembros del Opus Dei está afectada por una especie de sideología dualmente decidida"; por un lado, el miembro puede jactarse de trabajar por mejorar la situación de la comunidad movido por una ideología altruista y, por el otro, se puede luchar arduamente en pos del éxito personal. Además, el perfeccionamiento del trabajo de la élite intelectual del Opus Dei requiere estudio científico, y es precisamente aquí que encuentro el argumento principal para suponer que la Escuela de Alcalá estuvo bajo la influencia de dicha Institución. En un trabajo previo he denominado el concepto de la ciencia y la tecnología dentro del Opus Dei, el positivismo instrumentalista guiado por la élite intelectual católica, por "positivismo teo-tecnocrático. ${ }^{53}$. Esta denominación se refiere al hecho de que aunque el Opus Dei puede ser considerado como estrictamente conservador en materia de moral, sus miembros frecuentemente ponderan las bondades de la ciencia y la tecnología. Este 'espíritu modernista' parece ser la característica más sobresaliente de la orden, lo culal no puede entenderse correctamente si no se tiene en cuenta su motivación religiosa. En la visión del Opus Dei, la ciencia y la tecnología adquieren una importancia capital como medio efectivo de alcanzar los objetivos religiosos, ya que cumplirían el rol de instrumentos para la creación de una sociedad católica próspera y homogénea. No obstante, se pone énfasis en que esta actividad debe estar subordinada a valores espirituales más elevados $y$, por lo tanto, se postula el rol de la élite intelectual guiada por los valores morales del Opus Dei.

La reforma administrativa se caracteriza por su receptividad a la ciencia y la tecnología administrativas, y en cierta medida la manipulación del trabajo en la Administración pública de manera que ésta esté al servicio del desarrollo económico. Por lo tanto, creo que gran parte del tremendo esfuerzo hecho a partir de la Escuela de Alcalá con el objeto de cambiar el espíritu y el funcionamiento de la Administración pública espanola se debió en buena medida a la influencia de la ética profesional del Opus Dei, que sostiene que la tarea apostólica es rendir al máximo en la profesión particular de cada uno para así contribuir al desarrollo de una gran comunidad católica integrada. Aparte del hecho de que muchos de los actores centrales de la Escuela eran miembros del Opus Dei, la orientación intelectual de la Escuela converge con los postulados del Opus Dei en cuanto a ciencia y tecnología. Como este concepto se contrapone a la concepción tradicional española, debo concluir que la Escuela de Administración de Alcalá de Henares sólo puede interpretarse correctamente teniendo en cuenta la influencia del Opus Dei.

\section{Efectos de la reforma}

Se han hecho varios estudios sobre los efectos de la reforma tecnocrática de la Administración ${ }^{54}$. La mayoría de los mismos se relacionan de alguna manera con el estudio de Juan Linz y Amando DE MIGUEL, La élite burocrática española ante la reforma administrativa, basado en un estudio realizado entre los funcionarios superiores en 1967, y realizado por un grupo de sociólogos y expertos en Administración pública, pedido y financiado por la Escuela Nacional de Administración Pública ${ }^{55}$.

En mi estudio el punto principal ha sido: ¿De qué manera afectó la reforma en la mentalidad de los funcionarios públicos y en qué medida esto se tradujo en su trabajo? Éste es un aspecto que queda un poco en segundo plano en los estudios mencionados arriba, lo que puede explicarse por el hecho de que estos estudios se llevaron a cabo en un contexto en que las 
discrepancias entre los ideales y la realidad eran realmente llamativas. Además, aquellos que llevaron a cabo estos estudios eran decididamente críticos tanto de las tendencias manipuladoras de los reformistas tecnócratas como de los rasgos tradicionales de la Administración pública española. Como tales, pienso que tienden a poner el énfasis en los aspectos del trabajo administrativo que presentaban características reminiscentes del pasado histórico. Entonces, sería lógico preguntarse: ¿Qué falló? Basándome en el estudio de 1967 como asimismo en los estudios posteriores al respecto, yo preferiría reformular la pregunta de una manera que quede de forma más constructiva: ¿Qué se consiguió? Haciendo esto, es posible modificar y concretizar la crítica de los efectos de la reforma. Por lo demás, los estudios consultados también consideran que se produjeron importantes cambios a lo largo del proceso:

En la conclusión de su estudio, LINZ y DE MIGuEL escriben:

•.... Jcreemos que cualquier intento de reforma administrativa no llegará al término propuesto hasta tanto otras reformas de tipo educativo den sus frutos y produzcan un cambio de mentalidad. Es posible que incluso uno de los mecanismos de ese cambio se produzca como resultado de algunos éxitos parciales de la misma reforma administrativa ${ }^{56}$."

Luego, en 1977, BeLtrán afirma que;

-Parece, en conjunto, que se ha superado una época en la que el cuerpo era el único elemento estructural concebible para la organización de la función pública; no quiere esto decir en modo alguno que esa peculiar característica de la Administración española haya desaparecido, pero si que ha sufrido los embates del tiempo, y que los rasgos de feudalismo y estamentalidad que implicaba han dejado de alguna manera de ser posibles hoy en día, o al menos no de manera tan descarnada como lo fueron no demasiados años atrás ${ }^{57}$."

Y finalmente NIETO dice en su artículo de 1976 que;

La reforma, por descontado, no cumplió ni remotamente sus objetivos, fracasando en todos y cada uno de ellos y defraudando de manera lastimosa las esperanzas de la opinión pública $y$, sobre todo, de los propios funcionarios; pero sería injusto no reconocer que fue el primer intento serio -parcialmente logrado- de adaptar la Administración española a las transformaciones económicas y sociales, mejorando sustancialmente su imagen y mentalidad. A partir de esta reforma la Administración está ya dispuesta a asumir la responsabilidad de una fuerte intervención pública en la vida social y un cierto dirigismo económico; lo que aún no está en condiciones de hacer, evidentemente, y ésa es su tragedia, pero al menos ya no esquiva ni la tarea ni la responsabilidad. [. . I Posiblemente una de las consecuencias más trascendentales -aunque no sea la más llamativa a corto plazo- de la reforma haya sido cabalmente el romper la rigida estructura cor-

\section{porativa de la Administración, asi como la rigidez jerárqui-} ca existente de cada Cuerpo ${ }^{58}$."

Con objeto de modificar la crítica debo remontarme a mi división inicial de la reforma en tres esferas: la legal, la funcional y la cultural:

El aspecto legal tenía como objetivos la especialización, la desconcentración y la centralización ejecutiva. Esto estaba lejos de conseguirse durante este periodo. Aunque la Administración se transformó legalmente en una entidad, las leyes apenas podían alcanzar aquellos Cuerpos establecidos por -leyes especiales". Esta parte de la reforma podía tener éxito sólo si los Cuerpos generales arrebataban el poder dentro de cada Ministerio pero el grado en que esto se produjo fue muy irregular. $E l$ aspecto fincional de la reforma se refería a la manera de realizar el trabajo en la Administración y en el informe de 1967 se revela como el aspecto más impopular de la reforma ${ }^{59}$. Se puede suponer que esto fue así debido a que alteró la rutina acostumbrada del trabajo diario. No obstante, a pesar de su acogida poco favorable, las solicitudes para los cursos de Organización y Métodos fueron seis veces más numerosas que las plazas que pudieron ofrecerse en la Escuela de Alcalá en $1965^{60}$.

Estos dos aspectos afectaron directamente el trabajo diario y la lucha por el poder dentro de la Administración. Teniendo en cuenta el poder del sistema descoordinado y particularista, no sorprende a nadie el hecho de que una reforma administrativa gestada en el seno de la intelectualidad no pudo vencer la resistencia que encontró durante diez años. A medida que la reforma se llevó a cabo el resultado fue más bien la coexistencia de lo antiguo y lo nuevo. En algunos Ministerios, sobre todo los Ministerios económicos, el impacto del anuevo estilo fue significativo. No obstante, nunca se produjo un simple reemplazo de paradigmas administrativos. Cuando los "Cuerpos especiales* y sus métodos tradicionales se sintieron atacados, reaccionaron reduciendo sus servicios ${ }^{61}$. Los •burócratas de nuevo cuño (NIETO) seguramente hicieron sentir su influencia, pero ésta se limitó no obstante a aquellas áreas que no habían pertenecido a los poderosos .Cuerpos especiales. Y y ya que éstos, como estrategia defensiva, prácticamente boicotearon la reforma, la burocracia comenzó a disolverse. En palabras de NIETO:

“Durante la década de 1960 la Administración española experimenta un proceso de transformación que es de invertebramiento. Los Cuerpos de funcionarios, que habian sido siempre su columna vertebral, pasan a segundo plano. De esta manera la Administración se hace más ágil, pero, por otro, y a falta de un respaldo que le dé consistencia, no puede resistir los ataques de una política disfrazada. La época en que suele entenderse que la Administración alcanza su apogeo, e invade incluso el campo político, es en realidad -y quizá por eso- en la que empieza su disolución ${ }^{62}$." 
El aspecto cultural de la reforma trató de infundir a los funcionarios civiles un espíritu más universalista y propenso al cambio a la vez que intentó romper la rutina jurídica formal. Esto se buscó mediante el impacto producido por el desempeño de los funcionarios educados en la Escuela de Alcalá, lo cual parece haber sido la parte más exitosa de la reforma. En el informe de 1967 sólo el 20\% de los encuestados estimaron que la ley era un conocimiento indispensable o el más importante para un funcionario público, y casi el 50\% prefería reducir el impacto del conocimiento jurídico. El 69\% definieron que el criterio básico de éxito para su carrera era "conseguir una sólida reputación cientifican, y el 70\% consideraron que el criterio universalista para la promoción predominaba dentro de la Administración pública ".3. Esto podría explicarse por el hecho de que éste fue el elemento más "blando" de la reforma y que las personas que contestaban dijeron más o menos lo que se esperaba. No obstante, como afirman Linz y De Miguel, esto también puede interpretarse como un primer signo de cambio de mentalidad. Aparte de esto, y mucho más importante, pienso que el notable apoyo obtenido por los ideales universalistas expresado en el informe implicaba una legitimación de una nueva manera de concebir, y consecuentemente realizar, el trabajo administrativo.

\section{Reforma tecnocrática y transición democrática}

La reforma administrativa tecnocrática estaba íntimamente ligada con la totalidad de lo que puede considerarse el conjunto de reformas tecnocráticas del período, lo cual abarcaría, así pues, aspectos de la economía y la educación. La reforma administrativa como tal no sólo afectó la sociedad mediante el cambio directo en el trabajo de la Administración pública sino también a través de los cambios en las políticas económicas y educativas. En contraste con la retórica tradicional del régimen franquista, la cual, por así decirlo, hablaba en pretérito para justificarse a sí misma, los tecnócratas enfatizaban las posibilidades de futuro, tanto para los individuos como para el conjunto de la sociedad, haciendo de ello la justificación del régimen. El objetivo primario del Estado era ahora crear riqueza, elevar el nivel de vida y proporcionar 'igualdad de oportunidades' por medio de la educación científica y tecnológica, siempre intentando minimalizar la relevancia de la ideología en general. Es obvio que éste era un fundamento mucho más prosaico para una dictadura autoritaria que la vieja retórica belicista tan característica de Franco y los políticos tradicionales del régimen.
Además, uno de los problemas españoles más recurrentes de los últimos dos siglos había sido encontrar respuestas a los problemas derivados del desarrollo de una sociedad industrial moderna, y los sectores católicos se habían mostrado muy escépticos ante cualquier tendencia que podía conducir a una sociedad moderna en cuya construcción la ciencia social jugara un rol instrumental. A mi juicio, una parte importante del rol histórico de la reforma administrativa de los años sesenta será el ser el introductor de una tradición administrativa completamente nueva en España. Personas asociadas con el Opus Dei insertaron la ideología del management norteamericano en la España franquista a través de una ética religiosa marcadamente elitista, y de esta manera un aspecto del americanismo fue vertido dentro del molde del elitismo de la tradición católica española, con anterioridad reacia a toda "cientifización" de la sociedad. La Escuela de Alcalá se transformó en el lugar donde se produjo esta cientifización. de la función pública española. A pesar de todo, creo que la ciencia y la tecnología importada cobraron otro sentido dentro de la sociedad franquista tardía con el paso del tiempo. Esta interpretación se ve reforzada por el hecho de que todos los estudios sobre los efectos de la reforma administrativa llevados a cabo hacia fines de la década del sesenta y durante la del setenta alaban los aspectos universalistas y objetivos de la ciencia, considerando este ideal como uno de los prerrequisitos para la liberalización dentro del Estado español. Como tal, tanto LinZ, DE Miguel, Beltrán, NiETo y Carlos Moya pueden ejemplificar lo que quiero decir al afirmar que las ciencias sociales importadas cambian de significado con el correr del tiempo: la ideología universalista y meritocrática ya no sólo era parte de un proyecto político tecnocrático sino que además se podía convertir en la base de las reivindicaciones de desarrollo y avance sociales hechas por una creciente clase media, al promover lo que Moya llama reforma nacional liberal ${ }^{64}$. La ciencia tiene sus aspectos manipulativos como también anti-autoritarios. Por una parte, puede legitimar ciertas decisiones políticas como la "única manera. de hacer las cosas. Éste es, a mi juicio, el aspecto más notable de la fase inicial de la reforma administrativa. Por otro lado, la ciencia puede allanar el camino para la comunicación y el debate, cuando los argumentos se transforman en autoridad suprema.

La historia de la transición y consolidación democráticas en España no es la historia sobre una disolución continuista y sin conflictos del régimen franquista iniciada con la entrada en la arena política de los tecnócratas a finales de los años cincuenta. Los años que van de $1970^{\circ}$ a 1975 se caracterizaron por una, en mayor o menor medida, permanente crisis política interna del propio régimen. La transición a la democracia tras la muerte del dictador fue además negociada a través de acciones políticas de apoyo a la democracia que de ninguna forma estuvieron decididas de antemano. Sin embargo, tales acciones deben entenderse a la luz del desarrollo político, económico, social y cultural durante la última parte del régimen de Franco. Tal desarrollo se 
produjo bajo la influencia del sistema capitalista mundial en el contexto de la Guerra fría, y gracias a la labor de movimientos sociales nacionales, sindicatos y organizaciones políticas clandestinas que apoyaron una liberalización política. Un mayor entendimiento del desarrollo de esta etapa de la historia española debe, no obstante, completarse mediante la comprensión de cómo la llamada reforma administrativa tecnocrática influyó en los procedimientos políticos y en la cultura política e intelectual del país. Por lo tanto, creo que es justo decir que la reforma administrativa facilitó la subsiguiente transición a la democracia, pero que esto fue asi en principio como consecuencia de los siguientes tres factores no intencionados, íntimamente conecta- dos entre sí: 1) Apoyó un desarrollo económico y cultural que erosionó las bases sobre las que descansaba el régimen. 2) Contribuyó directamente a la disolución de una Administración española particularista y descoordinada que administraba y se repartía el poder dentro de la coalición franquista y 3) legitimó una nueva manera de concebir y realizar el trabajo en la Administración que se acomodaba a los intereses de una clase media urbana educada y siempre en crecimiento. Como tal, la reforma administrativa tecnocrática es uno de los factores clave a tener en cuenta a la hora de entender el carácter relativamente sosegado de la transición a la democracia en España al final de los años setenta.
- Departamento de Historia. Universidad de Oslo.

1 Este articulo se basa en mi resina de historia entregada en la Universidad de Oslo en la primavem de 1998: Modemization and Administrative Reform during the later Iranquist Regime (1957-1973) - Opus Dei professionalism and Spanish European lntegration.

2 Citado de losé V. Casinovn (1983), The Opus Dei Ethic and the Modernization of Spain, Ph.D. diss., New School for Social Research. Ann Arbor, Michigan: University Microfilms International, p. 284. (Irad. mío)

" Laureano Lóll:: Rono (1963a), La administración pliblica y las transformaciones socioeconomicas, Imprenta Nacional del Bolelín Oficial del Estado, pp. 93-95.

- Op.cit., p. 82.

'Laureano Loll:. Rono (1963b), •Administración pública y desarrollo económico en Documentación Administratima n. 65:1963, p. 17.

- El uso del lérmino recnócrata. tiene que ser calificado. Ha sido una crílica recurrente de los llamados tecnócratas españoles que eran más políicos que técnicos, o con las palabms de Alejandro Ni:io epolíicos disfrazados. Pienso que esio en parte es verdad. Las reformas cientamente formaron parte de un proyecto politico, pero rodo proyecto recnocrítico es por naturaleza político ya que imata sobre formas de hacer politica. Si las reformas emn tecnocráticas o no trata de técnicas de tomar decisiones, y no de si tenía motivaciónes políticas o no. Pienso que una de las caracteristicas más notables de un tecnócrata es que va a reivindicar que hasta una decisión politica tiene que estar hecha de manera cientifica. Pienso que esto es exactamente lo que fue propuesto por los tecnócratas españoles como López Rodó, Navarro Rubio, Ullasires y aquellos que, desde dentro de tanto el gobierno como la Adiministración, identificaron sus intereses en construir un Estado moderno basado en una Administración para el desarrollo económico.

7 Laureano LOHi:z ROIX (1990), Memorias (Vol.1), Barcelona, p. 64.

- Cindo de Casanova, op.cit., p. 290. (trad. mío).

${ }^{2}$ Ley de Régimen Juridico de la Adminisıración del Estado (31 de julio de 1957) y Ley de Procedimiento Administrativo (18 de julio de 1958).

I' Descle el 21 de julio de 1966 el Centro llevaba el nombre Escuela Nacional de Administración Priblica, y más tarde, el 30 de marzo de 1977 obtuvo el nombre que to davia liene, Instituto Nacional de Administracion Priblica.

"1960-70. Crónica de 10 años, Escuela Nacional de Administración Pública, Alcalá de Henares, p. 27.

12 Memoria de la Secretaria General Técnica correspondiente al año 1958, Presidencia del Gobierno, Madrid, p. 70, y Memoria de la Secretaria General Técnica. Año 1959, Presidencia del Gobierno, Madrid, p. 59.

is 1960-70: Crónica de 10 anios, op.cit., p. 27.

14 Menoria de la secretaria General Técnica comespondiente al año 1958, op.cit., p. 67. is Memoria de la Secretaria General Técnica: Año 1960, Presidencia del Gobierno, Madrid, p. 65.

${ }^{16}$ Miguel Bril.jrin (1994), Politica y administración bajo el franquismo: Ln reforma administrativa y los planes de desarollo, Working Paper 53/1994, Instituto Juan Narch de Esrudios e Investigaciones, Centro de Esrudios Avanzados en Ciencias Sociales, Madrid, p. 14.

17 Laureano LOPE: RODo (1958), La reforma administrativa en España. en Documenunción Administrativa n. 1:1958, p. 14.

18 Memoria de la Secretaria General Técnica. Año 1959, op.cit., p. 39.

19 Rostow propone en su teoria que todas las sociedades pasan por cinco etapas de desarrollo económico: la sociedad indicional, las precondiciones para el despegue, despegue, el camino a la madurez y la edad del alıo consumo de masa. (Jorge Li. RRAN (1989), Theories of Detelopmenl, Polity Press, Canbridge, UK, p. 96).

"LARRAN, op.cit., p. 97.

"Ciclos de conferencians sobre politica y planes de la Administración del Estado, problemas politicos y sociales de acmalidad y estudios superiores de Derecbo administrati1.0 que fueron pronunciados al II Curso de Técnicos de Administración Cinil. Documento del Centro de Formación y Perfeccionamiento de Funcionarios, Alcalá de Henares, 1963.

"L Laureano Lorl:: RoDo (1963a), op.cit., pp. 125-126.

${ }^{23}$. Oposiciones y concursos. en Boletin Oficial del Estado, 19 de mayo de 1960.

24 Memoria del año 1961, Centro de Formación y Perfeccionamiento de Funcionarios, Alcalá de Henares, p. 12.

x Miguel Br:Ltrin (1977), La élite burocrática española, Fundación Juan March, Editorial Ariel, Madrid, p. 147.

26 Op.cit., p. 150.

27 Plan de Estudios para el I curso de técnicos de Administarción civil. Alcalá de Henares, 1960. Documento del MAP, Madrid, p. 4.

2 Ciclos de conferencias sobre política y planes de la Administración del Estado, problemas políticos y sociales de actulalidad y estudios superiores de Derecbo administrali1.0 que fueron pronunciados al II curso de Técnicos de Administración Ciuil, op.cit., pp. 1-3.

2. Memoria del curso 1963-64, Centro de Formación y Perfeccionamiento de Funcionarios, Alcalá de Henares, p. 41.

30 Op. cit., pp. 42-44.

${ }^{31}$ Op. cit., pp. 44-46.

32 Op. cit., p. 49.

Op. cit., pp. 50-51.

Op. cit., pp. 52-53. 
3 Op. cil., p. 56.

${ }^{36}$ Op. cil., pp. 56-57.

${ }^{7}$ Op. cit., pp. 47-49.

Op. cil., pp. 57.59.

3) Op. cit., p. 59.

"1960-70: Crónica de 10 años, op.cit., p. 16.

"Op.cit., p. 4.

12 Op.cit., p. 39.

4. Op.cit., p. 32.37.

"Op.cit., p. 24

15 Cisinovi, op.cit., p. 232.

16 1960-70: Crónica de 10 arios, op.cit., p. 39

17 Op.cit., p. 19.

${ }^{48}$ Op.cit., p. 24 ) 26.

${ }^{49}$ Casinovi, op.cit., p. 159.

51) Joan Es7RuCil (1995), Saints and Schemers: Opus Dei and Its Paradoxes, New York, Oxford University Press, p. 250. (trad. mio).

$"$ Citado de Casanovi, op.cit., p. 92. (trad. mio).

s. Michael Wilsil (1989), The secret urorld of Opris Dei, Grafion Books, London- Glasgow-Toronto-Sydney-Auckland, p. 144 (trad. mío).

"sobre el concepto de la ciencia y' la tecnologia dentro del Opus Dei, véase H:LLnG (1998), pp. 58-86.

* Los estudios aludidos aquí y revisados en mi trabajo, Modenization and Administrative Reform during the Inter Franquist Regime (1957-1973), son: Miguel BrLtRiN (1977) Ln élite burocrática espaniola, Fundación Juan March, Editorial Ariel, Madrid. Migeul Be:Lrin (1996), La Adininistración. en losé Maria Jovi:k Zamora (ed.), Historia de Esparia, MEN(ENI):. PIIDAL. La época de Franco (1939-1975). Vol I , Espasa Calpe S.A., Madrid. Miguel Be:LrRin (1994), Politica y administración bajo el franquismo: La reforma administrativa y los planes de desarollo, Working Paper 53/1994, Instituto Juan March de Estudios e Investigaciones, Centro de Estudios Avanzados en Ciencias
Sociales, Madrid. Juan I. LIN\%, Amando DE: Micur:L (1968), La élite funcionarial espanola ante la reforma administrativa. en Luis S.incill:z. AGlsin (ed.), Sociologia de la Administración Priblica Espaniola, Centro de Estudios Sociales de la Santa Cruz del Valle de los Caidos, Madrid. Alejandro Ni:10 (1976), .De la República a la Democracia: la Administración espanola del franquismo (I). en Cititas. Rerista Espaniola de Derecho Administrativo, n. 11 y Alejandro Nitio (1977), .De la República a la Democracia: la Administración espanola del franquismo (II). en Cinitas. Rerisla Espariola de Derecbo Administrativo, n. 15.

"Bs:L7kin (1977), op. cit., p. 7. Los investigadores que condujeron el estudio fueron: F. Andrés OrIzo, Francisco ANSON, Miguel Bilitrin, Luis Fernando CRlsiro, J. Julio

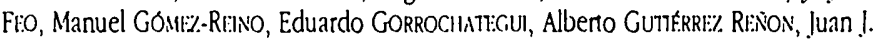

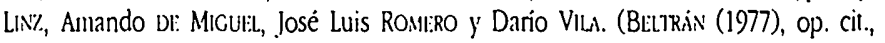
nola a pie de página 32, p. 27).

* Juan J. LIN\%, Amando DE MIGUl: (1968), La élite funcionarial española ante la reforma administrativa en Luis Sincill:: AGt:sTh (ed.), Sociologin de la Administración Priblica Espaniola, Centro de Estudios Sociales de la Santa Cruz del Valle de los Caidos, Madrid, p. 248.

57 Bl:L7kin (1977) op.cit., p. 123.

38 Alejandro Nil:1o (1976), . De la República a la Democracia: la Adminisiración espanoola del franquismo (I), en Civilas. Revista Espanola de Derecbo Administratimo, n. 11, p. 576. (cursiva mía).

\$9 LIN//De MiGUilL, op. cil., p. 236.

a Formación de especialistas de O.\& M. en la Administración pública española en Revista Internacional de Ciencias Administratitias, Vol XXXI - 1965, n. 1, International Institute of Administrative Science, Bruxelles. Articulo del Centro de Fommación y Perfeccionamiento de Funcionarios, p. 45.

G Alejandro Ni:ıo (1977), -De la República a la Democracia: la Administración espanòla del franquismo (II). en Citritas. Revista Espaniola de Derecho Administratiro, n. 15, p. 579 .

w NI:IO (1976), op.cit., pp. 577-578. (tmd. mio).

6. Sobre la mentalidad expresada por los funcionarios en la encuesla de 1967, ver

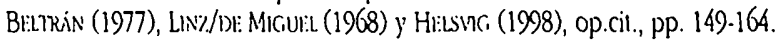

a Ver Bl:L7rin (1977), op.cit., p. 27.
Bl:L7kin, Miguel (1977), La élite burocrática espariola, Fundación Juan March, Editorial Ariel, Madrid.

Bt:LrRin, Miguel (1994), Politica y administración bajo el franquismo: La reforma administrativa y los planes de desarrollo, Working Paper 53/1994, Instituto Juan March de Estudios e Investigaciones, Centro de Estudios Avanzados en Ciencias Sociales, Madrid.

Bl:Ltrin, Miguel (1996), La Administración. en JOV:R Zasiori, José Maria (ed.) Histo. ria de Esparia, ML:NE:NDl: PIIIAL. La época de Franco (1939-1975). Vol I , Espasa Calpe S.A.

Castinova, José V. (1983), The Opus Dei Ethic and the Modemization of Spain, Ph.D. diss., New School for Social Research. Ann Arbor, Michigan: University Microfilms In. ternational.

Ciclos de conferencias sobre política y planes de la Administración del Estado, problemas politicos y sociales de actualidad y estudios superiores de Derecho adminisrativo que fueron pronunciados al Il Curso de Técnicos de Administración Civil. Documento del Centro de Formación y Perfeccionamiento de Funcionarios, Alcalá de Henares, 1963.

1960-70: Crónica de 10 años, Escuela Nacional de Administración Pública, Alcalá de Henares.
EstruCII, Joan (1995), Saints and Schemers: Opus Dei and Its Paradoxes, New York, Oxford University Press.

-Formación de especialistas de O. \& M. en la Administración pública espaniola en Revista Intemacional de Ciencias Administrativas, Vol XXX] - 1965, n.1, International Institute of Administrative Science, Bruxelles. Articulo del Centro de Formacion y Perfeccionamiento de Funcionarios.

Helsvg, Kinl (1998), Modemization and Administrative Reform during the later Franquist Regime (1957-1973) - Opus Dei professionalism and Spanish European Integration, Universidad de Oslo.

Lakrin, Jorge (1989), Theories of Detelopment, Polity Press, Cambridge, UK.

LINZ, Juan J./DE MIGUF:L, Amando (1968), La élite funcionarial espanola ante la refortha administrativa. en Sincill:. AGl:sTA, Luis (ed.), Sociologia de la Administración Ptiblica Espaniola, Centro de Estudios Sociales de la Santa Cruz del Valle de los Caidos, Madrid.

Lóprz Rodó, Laureano (1958), -La reforma adninistrativa en España• en Documentación Administrativa n. 1:1958.

LOPF.7. RODÓ, Laureano (1963a), La administración priblica y las transfonmaciones socioeconómicas, Imprenta Nacional del Boletin Oficial del Estado. 
Lóli: Ronó, Laureano (1963b) AAduninisıración puiblica y desarrollo económico. en Docimmentación Administratima n. 65:1963.

Lólv: Rono, Laureano (1990), Memorias (Vol.1), Barcelona.

Memorin de In Secretaria General Técnica correspondiente al ano 1958, Presidencia del Gobicrno, Madricl.

Memoria de In Secretarin General Técnica: Aino 1959, Presidencia del Cobierno, Madricl.

Memorin de In Secresaria General Tëcnica: Ano 1960, Presidencia del Cobierno, Nacricl.

Memoria del ario 1961, Centro de Formación y: Perfeccionamiento de Funcionarios, Alcalí de Henares.
Memoria del curso 1963-64, Ceniro de Formación y Perfeccionamiento de Funcionarios, Alcalá de Henares.

Nu:10, Alejandro (1976), .De la República a la Democracia: la Adminisıración espanola del franquismo (1). en Ciritas. Rerista Espaniola de Derecbo Administratitio, n. 11.

Nı:to, Alejandro (1977), •De la República a la Democracia: la Administración espanola del franquismo (II) en Civitas. Revista Espaniola de Derecbo Administrativo, n. 15.

Oposiciones y concursos. en Boletin Oficial del Estado, 19 de mayo de 1960.

Plan de Estudios para el I Curso de Técnicos de Administarción Civil. Alcalá de Henares, 1960. Documento del MAP, Madrid.

WMsil, Michael (1989), The secret world of Opus Dei, Grafton Books, London-Glasgow-Toronto-Sy:dney-Auckland. 
